\title{
Video Article \\ Middle Cerebral Artery Occlusion Allowing Reperfusion via Common Carotid Artery Repair in Mice
}

\author{
Melissa Trotman-Lucas ${ }^{1}$, Michael E. Kelly ${ }^{2}$, Justyna Janus ${ }^{2}$, Claire L. Gibson ${ }^{1,3}$ \\ ${ }^{1}$ Department of Neuroscience, Psychology and Behaviour, University of Leicester \\ ${ }^{2}$ Preclinical Imaging Facility, Core Biotechnology Services, University of Leicester \\ ${ }^{3}$ School of Psychology, University of Nottingham
}

Correspondence to: Claire L. Gibson at claire.gibson@nottingham.ac.uk

URL: https://www.jove.com/video/58191

DOI: doi:10.3791/58191

Keywords: Medicine, Issue 143, Neuroscience, stroke, transient MCAO, brain, ischemia, middle cerebral artery

Date Published: 1/23/2019

Citation: Trotman-Lucas, M., Kelly, M.E., Janus, J., Gibson, C.L. Middle Cerebral Artery Occlusion Allowing Reperfusion via Common Carotid Artery Repair in Mice. J. Vis. Exp. (143), e58191, doi:10.3791/58191 (2019).

\section{Abstract}

The ischemic stroke is a major cause of adult long-term disability and death worldwide. The current treatments available are limited, with only tissue plasminogen activator (tPA) as an approved drug treatment to target ischemic strokes. Current research in the field of ischemic stroke focuses on better understanding the pathophysiology of stroke, to develop and investigate novel pharmaceutical targets. Reliable experimental stroke models are crucial for the progression of potential treatments. The middle cerebral artery occlusion (MCAO) model is clinically relevant and the most frequently used surgical model of ischemic stroke in rodents. However, the outcomes of this model, such as lesion volume, are associated with high levels of variability, particularly in mice. The alternative MCAO model described here allows the reperfusion of the common carotid artery (CCA) and the increased perfusion of the middle cerebral artery (MCA) territory, using a tissue pad with fibrinogen-based sealant to repair the vessel, and the improved welfare of the mice by avoiding external carotid artery (ECA) ligation. This reduces the reliance on the Circle of Willis, which is known to be highly anatomically variable in mice. Representative data show that using this alternative surgical approach decreases the variability in lesion volumes between the traditional MCAO approach and the alternative approach described here.

\section{Video Link}

The video component of this article can be found at https://www.jove.com/video/58191/

\section{Introduction}

A major cause of cerebral stroke is focal ischemia in the territory of the middle cerebral artery. Tissue plasminogen activator (tPA) is the only pharmacological treatment available with proven efficacy, despite numerous clinical drug trials targeted to ischemic stroke ${ }^{1,2}$. However, due to safety concerns and a narrow therapeutic window $(<4.5 \mathrm{~h})$, only $\sim 15 \%$ of all stroke patients are eligible to receive tPA, and the recanalization rates can be $<50 \%{ }^{3,4}$.

Reproducible and clinically relevant animal models of stroke are considered essential to inform the development of new and potential stroke therapeutic treatments. However, due to concerns regarding consistency and variability in the outcomes with animal models, it remains important to refine existing in vivo models to improve the translation from preclinical studies to the clinic. The lack of translation from the preclinical experimental efficacy of potential treatments to clinical use is an ongoing concern for stroke research ${ }^{5}$. Reasons for failure of translation are likely to be multiple and may be related to, for example, the trial design, treatment delays, clinical stroke heterogeneity, and the limitations of the animal models used ${ }^{6}$. A key challenge for stroke research remains the development of safe and effective treatments.

Middle cerebral artery occlusion (MCAO) by intraluminal filament insertion is the most frequently used in vivo rodent model of experimental stroke. This model allows the restoration of the blood flow after an ischemia induction, mimicking the events that occur in human stroke ${ }^{7}$. However, in particular in mice, heterogeneous lesion volumes with varied standard deviations occur even though defined surgical protocols are applied ${ }^{8,9,10}$. It is typical to see a bimodal distribution of small striatal and large striato-cortical lesion volumes ${ }^{11}$. To induce ischemia, the filament is typically inserted through an incision of the CCA or ECA which then remain permanently ligated ${ }^{12}$. The permanent ligation of the CCA prevents the re-establishment of the blood flow into the internal carotid artery (ICA) and, subsequently, the MCA territory. This causes reperfusion to be reliant on the collateral supply within the Circle of Willis (CoW). The CoW structure has anatomical variability between individual animals, particularly in C57BL/6 mice-a strain typically used in in vivo stroke research ${ }^{13}$. An alternative method, of filament insertion through the ECA, does allow the continued perfusion through the CCA, but this method compromises the arterial supply to the ECA territory, which has been shown, in rats, to have a detrimental effect on the animal's well-being ${ }^{14}$.

The reliance on the CoW for collateral supply and reperfusion in the established MCAO model may, in part, account for the lesion volume variability following occlusion. We describe an alternative murine surgical procedure where ECA ligation is avoided and the CCA incision is repaired, thus permitting reperfusion through the CCA, independent of the CoW. The repair of the CCA incision has been previously shown in 
rats to result in a successful reperfusion through the $\mathrm{CCA}^{15}$. We have applied this approach successfully in mice ${ }^{11}$ and report here the protocol which results in a reduced variability in lesion volume, the main outcome measure used in experimental stroke studies.

In this protocol, we demonstrate how to undertake MCAO through CCA vessel filament insertion followed by CCA vessel repair, which involves a tissue pad and sealant application to permit reperfusion.

\section{Protocol}

This protocol and the data reported were conducted in accordance with the UK Animals (Scientific Procedures) Act, 1986 (Project license 60/4315) and following institutional ethical approval. All experiments are reported in accordance with the Animal Research: Reporting of In Vivo Experiments (ARRIVE) guidelines ${ }^{16}$.

\section{Preparation}

1. Familiarize adult male C57BL/6 mice to post-operative care (e.g., environment, bedding, recovery foods) at least $48 \mathrm{~h}$ before the surgery. NOTE: Post-MCAO animals often have difficulty with eating and drinking following surgery.

1. To prevent excessive weight loss following surgery, acclimatize the animals to any post-MCAO diet by, for example, placing rehydration gel, gel food, and soaked/wet normal diet pellets directly onto the cage floor.

2. Change the cage bedding to post-operative bedding, such as white paper chip.

2. Sterilize all surgical tools before beginning the surgical set-up or procedures.

1. Do this by autoclaving the tools (with a minimum of $121^{\circ} \mathrm{C}, 15 \mathrm{psi}$, for $15 \mathrm{~min}$ ) or through the use of ethylene oxide (following the correct manufacturer's instructions, for 8-10 h).

2. Disinfect all surfaces prior to setting up the procedure. Cover all surfaces with sterile surgical drapes or autoclaved foil for items that require handling during the surgery. Use aseptic technique for the duration of the procedure.

NOTE: Autoclaved foil can be used to cover the instruments or equipment that need to be held during the surgery. Using sterile gloves and techniques, the foil can be applied and, then, the item can be placed into the sterile field. Following this, a sterile glove change will be required.

\section{Middle Cerebral Artery Occlusion Surgery}

1. Use adult male C57BL/6 mice weighing $24-31 \mathrm{~g}$ at the time of the surgery. Induce anesthesia using $5 \%$ isoflurane in $2 \mathrm{~L} / \mathrm{min}$ of $\mathrm{O}_{2}$, in a red plastic anesthesia chamber.

2. Following the induction, reduce the isoflurane to maintain an adequate anesthesia depth for the surgery $\left(\right.$ e.g., $1.5-2 \%$ in $70 \% \mathrm{~N}_{2} \mathrm{O}_{2} / 30 \%$ O $\left.\mathrm{O}_{2}\right)$, delivered by face mask combined with a scavenger system to lower the surgeon's exposure to isoflurane.

NOTE: Nitrous oxide $\left(\mathrm{N}_{2} \mathrm{O}_{2}\right)$ can be used in combination with oxygen $\left(\mathrm{O}_{2}\right)$ to reduce the required amount of isoflurane for an adequate anesthesia depth.

3. Administer systemic analgesia pre-operatively [carprofen, $10 \mathrm{mg} / \mathrm{kg}$ subcutaneous (sc.)] and local anesthesia to the incision site (bupivacaine, $2 \mathrm{mg} / \mathrm{kg}$ sc., diluted $1: 10$ in $0.9 \%$ saline) peri-operatively.

4. Administer fluids by intraperitoneal (ip.) injections of $200 \mu \mathrm{L}$ of sterile prewarmed $0.9 \% \mathrm{NaCl}$ solution pre-operatively.

5. Shave the fur of the right temporal region and ventral neck region using small hair clippers to expose the skin. Disinfect the surgical skin area, using a $5 \%$ chlorhexidine solution for $3 \mathrm{~min}$. Apply ocular lubricant onto both eyes, to prevent them from drying during the surgery. NOTE: If required for pulse oximetry readings to monitor blood oxygen saturation, heart rate, and breathing rate, use hair removal cream to clear the hind leg of hair. Applying the cream using clean cotton buds and, after the hair removal, wash the area with the dilute chlorhexidine solution. Perform this step in a pre-operative area to minimize the risk of loose fur contaminating the sterile surgical field. Researchers may prefer to use other surgical site preparations according to local practice.

6. Transfer the mouse to the surgical area and place it in the prone position on a homeothermic heat mat covered by a sterile drape. Maintain anesthesia via a nose cone. Insert a rectal probe to monitor the mouse's body temperature and maintain it at $37.0 \pm 0.6^{\circ} \mathrm{C}$.

1. Prior to any surgical incisions, check the hind paw withdrawal reflex and blink reflex to confirm the anesthesia depth.

7. Attach a laser Doppler flowmetry (LDF) probe to record the blood flow to the MCA territory.

1. Using a dissection stereoscope, make an incision of $<1 \mathrm{~cm}$ at the midpoint between the right eye and ear on the exposed temporal skin using a No. 15 scalpel. Bluntly dissect the underlying tissue covering the skull, gently scraping this away from the bone and drying the surface with sterile cotton swabs.

NOTE: Care must be taken not to damage the temporalis muscle.

2. Take hold of the $0.7 \mathrm{~mm}$, flexible single fiber-optic probe attached to the LDF monitor, cut its end using a sharp scalpel to gain a flat edge, push this through the donut-shaped probe holder, and place a small amount of optical matching gel at the end of the fiber.

3. Place a small amount of waterproof super-adhesive glue around the bottom edge of the probe holder; allow this to partially dry and become tacky.

4. Dry the skull above the temporal bone and place a small amount of topical tissue adhesive in a circle to the bone, just enough for the probe holder.

5. Place the probe holder, with the fiber optic probe already in place, on this area (6 $\mathrm{mm}$ lateral and $2 \mathrm{~mm}$ distal from the bregma).

6. Allow time for the glue to dry; once dry and attached, begin recording the LDF data.

8. Gently turn the animal over to a supine position, taking care to support the laser Doppler probe and prevent its detachment. Gently tape the two forepaws down with microporous medical tape, sliding a pair of cotton buds (or something similar, such as closed forceps) under the neck to lift the area and create tension. Cover the mouse with a sterile drape to maintain aseptic coverage. 
9. Begin the dissection and exposure of the CCA.

1. Make a $1.5 \mathrm{~cm}$ midline incision on the exposed ventral neck using a No. 15 scalpel blade.

2. Using gentle blunt dissection techniques, gently retract the salivary glands to the sides, exposing the trachea.

3. Using blunt dissection, dissect the CCA free from the surrounding tissue and vagal nerve.

NOTE: Avoid touching the vagal nerve directly, as any damage to the vagal nerve can impair mobility, feeding, and breathing.

10. Pass two small $(2 \mathrm{~cm})$ sections of a non-dissolvable 6-0 suture below the CCA, dorsal to the vessel and ventral to the vagal nerve. Draw one silk tie closer to the surgeon and tie it tightly around the CCA (proximal tie). Loosely tie the second silk tie (distal tie) toward the bifurcation of the ICA/ECA.

11. To begin to isolate a section of the CCA, apply a microvascular clip just above the distal tie but not obstructing the bifurcation. Using micro Vannas scissors, make a small hole into the CCA.

NOTE: The ECA must remain patent at all times. The CCA incision must be no more than $40 \%$ of the width of the vessel and in an easily accessible ventral position; this will play an important role in the vessel repair stage post-MCAO.

12. Insert a 7-0 silicone-coated monofilament into the CCA, advancing toward the microvascular clip.

NOTE: The filament size must be established based on the mouse's weight prior to the surgery; refer to the manufacturer's guidelines.

1. Tighten the distal tie, enough to fasten the filament into place without damaging it. Remove the microvascular clip using clip holders. Note: No blood loss should be seen at this point. If there is a backflow of blood, the tie holding the filament is not tight enough.

13. Advance the filament into the ICA.

1. Ensure the filament remains within the ICA and does not pass into the pterygopalatine artery (PPA). Do this by slightly lifting and pulling the CCA, using one of the silk ties, to the outer side of the animal's body, and steer the filament to bend past the internally facing opening of the PPA.

Note: Once advanced to the MCA branch origin, a drop in the relative blood flow to the supplied territory will be visible on the LDF values; this confirms the filament placement.

14. Secure the filament in place with the distal silk tie, tying this tighter. Leave it in place for the duration of the occlusion time period. NOTE: Dependent on individual protocols, the animal can be moved to a recovery cage, following wound suturing, to recover, or remain under anesthesia for the duration of the occlusion period. In the latter case, ensure the wound is prevented from drying out by using sterile prewarmed $0.9 \% \mathrm{NaCl}$ saline.

\section{Post-occlusion}

1. At the end of the MCAO period, immediately retract the filament until the white filament head is clearly visible.

2. Then, Loosen the distal CCA tie just enough to remove the filament head most of the way out of the vessel. NOTE: The filament can be fully removed at this point if the surgeon is confident with speed to tie the distal tie to prevent blood loss.

3. Place one microvascular clip in a horizontal position toward the CCA bifurcation next to the distal tie. Loosen the distal tie and remove the filament fully. Add another microvascular clip below the proximal CCA tie toward the surgeon.

NOTE: Ensure the vessel is far enough into the prongs of the clamp to prevent any slippage. The placement of the clips is essential to ensure clip removers easy access during later steps. The clips can be used to help lift the vessel to allow a better clearance and placement of the tissue patch, placing the clips horizontally across the surrounding muscles.

4. Carefully remove both silk ties using Dumont \#5 forceps or micro Vannas scissors and dry the area using sterile cotton buds. NOTE: Care must be taken not to cut through/into the vessel.

5. In a sterile Petri dish, add fibrinogen and thrombin sealant solutions 1 and 2 (see the Table of Materials), ensuring the two substances stay separate, ready for mixing later.

NOTE: Only very small volumes of the agents are required $(<0.25 \mathrm{~mL}$ each). Keeping the solutions separate prevents a premature reaction between the two constituents. Ensure the cap is replaced the same way as it was removed to prevent cross-contamination of the two syringes, which would cause the agent to react and set within the syringe. Prior to use, store the solutions at $-20^{\circ} \mathrm{C}$. When required for the first surgery, thaw the sealant to room temperature. Do not refreeze the sealant; it must remain at room temperature and can be stored this way. The syringe can be used across multiple surgeries making it cost-effective; however, we recommend to not use the same vial for longer than 1 week to prevent contamination.

6. Use blunt dissection along the muscle with Dumont no 5 forceps and micro Vannas scissors to obtain a thin ventral slice of sternocleidomastoid muscle to use for the tissue pad, ensuring the slice is no more than $1 \mathrm{~mm}$ thick and runs along the top fibers of the muscle.

NOTE: Do not cut through/across the muscle, as this will significantly impair its function. The tissue must be large enough to cover the CCA incision comfortably.

7. Using Dumont \#5 forceps, take the tissue pad and mix the tissue evenly across the two fibrinogen and thrombin sealant solutions, forming a channel between the two reagents. Coagulation will occur quickly; as soon as coagulation begins, remove the tissue pad to the CCA incision. Place the tissue pad flat down with a medium firm pressure and open forceps.

1. Swiftly remove the distal microvascular clip whilst still gently holding the tissue pad in place. Note: This allows some backflow of blood to further activate the fibrinogen and thrombin sealant reagents. Just enough pressure is required to hold the pad in place but not to fully block the vessel.

2. Slowly alleviate the pressure from the tissue pad, allowing blood to flow under the incision area. Now, slowly and gently release the pressure from the proximal microvascular clip and fully remove.

3. NOTE: In order to ensure the vessel becomes fully patent, the placement of the tissue pad and the removal of the microvascular clips must occur quickly to prevent the tissue pad from sealing to the inside of the CCA. However, if there is a small amount of blood leakage, re-place some light pressure onto the tissue pad to allow further time for clot formation and sealing to occur. If the blood leakage is substantial or the tissue pad does not appear to be sealing, maintain pressure to prevent blood loss and replace both CCA microvascular clips to isolate the incision and prevent further blood loss.

8. In the event the tissue pad does not seal to the vessel, a second attempt can be made, following steps 3.3-3.7.3. 
9. Once the vessel is sealed, suture the wound using dissolvable 6-0 sutures. If LDF recording was continued throughout surgery, remove the LDF from the skull and suture wound using dissolvable 6-0 suture.

\section{Post-operative Care}

1. Place the animal into a prewarmed recovery cage (situated on a heated shelf/mat at $35^{\circ} \mathrm{C}$, or within a heated chamber). NOTE: Researchers may prefer to use other temperatures and durations according to local practice.

2. Provide all animals with $200 \mu \mathrm{L}$ of prewarmed $0.9 \% \mathrm{NaCl}$ saline sc. immediately post-operation, at $4 \mathrm{~h}$ post-operation, and $2 \mathrm{x}$ daily for $72 \mathrm{~h}$. NOTE: The administration of prewarmed $0.9 \% \mathrm{NaCl}$ is animal led. If more is required, more fluids can be administered to ensure a good recovery.

3. In the recovery cage, give the animals unrestricted access to soggy diet pellets, dry diet pellets, rehydration gel, and gel food, alongside ad libitum access to water.

4. Repeat the sc. carprofen injection at $24 \mathrm{~h}$ post-operation (see step 2.3). NOTE: All animals received the same dose of carprofen; any neuroprotective effect is likely to be negligible.

5. At regular intervals for $48 \mathrm{~h}$, perform post-op mouse grimace scoring ${ }^{17}$ to assess pain levels to aid the decision to administer further analgesia.

6. Weigh the animals immediately before surgery and then daily following the procedure. Perform daily observations and complete welfare sheets to monitor their food and water intake and clinical signs.

7. Undertake functional observations at $24 \mathrm{~h}$ and $48 \mathrm{~h}$ post-operation. Assess the mice on a focal deficit scale. Evaluate their body symmetry, compulsory circling, gait, $45^{\circ}$ grid climbing, circling behavior, front limb asymmetry, and whisker touch response $e^{18,19,20}$.

\section{Magnetic Resonance Imaging and Image Processing}

1. Measure the lesion volume (LV) using structural magnetic resonance imaging (MRI).

NOTE: Alternative methods, such as histological staining with triphenyltetrazolium chloride (TTC), have previously been used and correlate to structural MRI data. However, this method can only be used at the endpoint of a study and not longitudinally. Using longitudinal MRI scanning will reduce the number of animals required for a study.

1. After $48 \mathrm{~h}$, following the induction of the MCAO, anesthetize the mouse with isoflurane ( $5 \%$ isoflurane in $1 \mathrm{~L} / \mathrm{min}$ of $\mathrm{O}_{2}$ for induction, $1.5-2 \%$ isoflurane for maintenance).

2. Transfer the mouse to the MRI cradle, place it on the respiration sensor for monitoring its breathing rate, and implant the rectal temperature probe for monitoring its temperature during the scanning. Place the 2-channel mouse-brain RF receive coil over the brain for a signal and place the cradle in a 9.4 T horizontal bore scanner.

NOTE: Here, a volume coil with a $72 \mathrm{~mm}$ inner diameter was used for the RF transmission.

3. Acquire T2-weighted scans using a fast spin-echo sequence. Set the repetition time (TR) to 3,000 ms and the echo time (TE) to 40 $\mathrm{ms}$. Use $18 \mathrm{~mm} \times 18 \mathrm{~mm}$ as the field of view (FOV), and obtain a $256 \times 256$ acquisition matrix with slices of $18 \mathrm{~mm} \times 0.8 \mathrm{~mm}$ and three signal averages in approximately $10 \mathrm{~min}$

4. Acquire diffusion tensor images (DTI) using a fast spin-echo sequence. Set the TR to $1,730 \mathrm{~ms}$, the TE to $35 \mathrm{~ms}$, the FOV to $20 \mathrm{~mm} x$ $20 \mathrm{~mm}$, and obtain a $128 \times 128$ acquisition matrix with slices of $16 \mathrm{~mm} \times 1 \mathrm{~mm}$, two signal averages, 14 diffusion encoding directions, and a maximum $b$-value of $1,024 \mathrm{~s} / \mathrm{mm}^{2}$.

5. Measure the LV on the T2-weighted images using an image display and measuring software package. Measure the lesioned area, collating the values together to calculate the total lesion volume while taking into account the MRI slice thickness (set during the MRI scan).

6. Take into account any swelling and the percentage of area lesion volumes while measuring the full contralateral and ipsilateral hemispheres. Correct for any brain swelling due to edema using an indirect method to measure the lesion volume as described previously ${ }^{21,22}$. Only include slices that contain cortex tissue and not frontal lobe or cerebellum tissue according to a standard mouse brain atlas to avoid overcorrection.

2. Measure the lesion diffusion parameters and obtain the core and penumbra regions of interest.

1. Generate the apparent diffusion coefficient $(A D C)$ and fractional anisotropy $(F A)$ maps from the diffusion tensor images using appropriate MRI analysis software.

2. Align the DTI images with the T2-weighted images using appropriate image analysis software capable of performing a linear registration of images. Following the registration, subtract the diffusion-weighted lesion masks (ischemic core) from the T2-weighted lesion masks (ischemic core and penumbra) to estimate the penumbra region. Apply the resultant masks (core and penumbra) to the $A D C$ and FA maps to quantify the diffusion parameters within the core and penumbra.

3. Translate the ipsilateral core and the penumbra masks about the brain midline in order to obtain contralateral ADC and FA values for comparison.

\section{Representative Results}

A total of 24 adult male C57BL/6 mice, weighing between $24-31 \mathrm{~g}$ at the time of surgery, were used in the study. One animal died following middle cerebral artery occlusion (MCAO) and one was excluded due to surgical complications. The data presented here are taken from a previously published work by the authors. These were used to illustrate the effect of vessel repair on MCAO outcomes ${ }^{11}$. All data are expressed as thr mean \pm standard deviation. The data were statistically assessed for normality using the D'Agostino-Pearson omnibus normality test. Parametric data were compared using Student's $t$-test (for two means) and one-way ANOVA with the Sidak test (multiple means). Nonparametric data were compared using the Mann-Whitney $U$ test. The variability of the parametric data was assessed using an $F$-test, and nonparametric data variability was assessed using Levene's test. 
Typically, in MCAO procedures, the occluding filament is inserted into the CCA and the ECA is ligated to prevent this filament from passing into the ECA rather than the ICA. An avoidance of ECA ligation and the addition of analgesia showed a trend toward reduced weight loss at $48 \mathrm{~h}$ post-MCAO, when compared to data from previous studies undertaken by the same surgeon for the same MCAO time using ECA ligation with no analgesia, whereas the LV appeared unaffected, see Figure 1.

Mice underwent a 60 min MCAO-induced ischemia followed by reperfusion with CCA vessel repair or with the typical ligation of the CCA approach. A schematic of the ligated and unligated repaired CCA is shown in Figure 2.

Laser Doppler flowmetry was used to confirm the blood flow perfusion in the territory of the MCA at MCAO, before and after the CCA vessel repair. Figure 3 demonstrates that $5 \mathrm{~min}$ following the filament removal, the regional cerebral blood flow (rCBF) significantly increased in the brain region of the MCA. The perfusion was maintained up to the vessel repair, with an increase in the perfusion to the MCA territory shown following the CCA vessel repair, suggesting that the CCA repair allowed an increased blood perfusion to the ischemic territory compared to reliance on the Circle of Willis alone.

T2-weighted MRI was used to determine the total LV, and DTI scans were used to determine the core LV, $48 \mathrm{~h}$ after the MCAO. Figure 4A shows no significant difference in the total or core LV between the repair and ligated procedure groups. However, the data variability for both total and core LV, as assessed using Lavene's test for non-parametric or the F-test for parametric data, was significantly reduced within the CCA repair group. The total LV was broken down into cortical and subcortical LV, as shown in Figure 4B. The cortical portion was significantly less variable in the CCA repair group, whereas the sub-cortical portion of the lesion was unaffected between the two procedural groups.

A power analysis indicated that fewer animals per treatment group would be required to demonstrate a $30 \%$ reduction in the LV following MCAO using CCA repair versus the typical CCA-ligated procedure, see Table 1. An assumption of power $1-\beta=0.8$ and significance level $\alpha=0.05$ and a prediction of $30 \%$ reduction in the LV between the hypothetical control and test groups were used for the power analysis. Furthermore, an equal variance was assumed between the groups. Table 1 shows the number of animals required for the test and control groups when either the typical CCA-ligated method is used or the updated CCA repair method, as described here, is used. Note that the test group refers to a hypothetical treated group of animals and the control group refers to a hypothetical control group; both groups would undergo MCAO. 
A

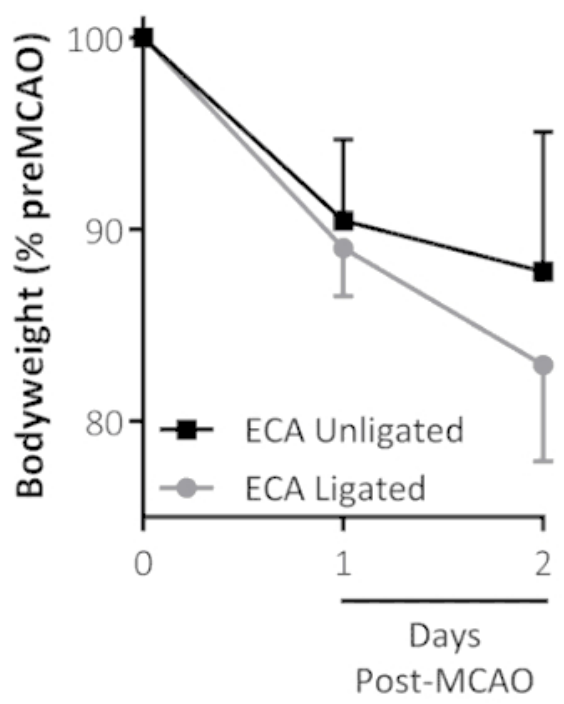

B

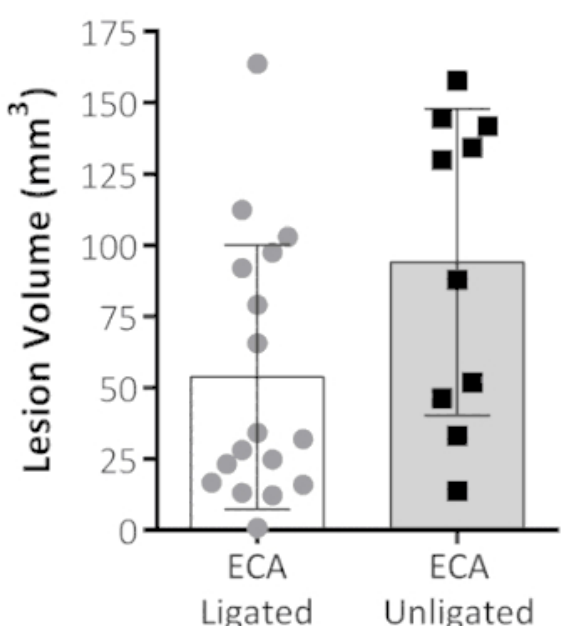

Figure 1: Combined analgesia treatment and the omission of ECA ligation on outcomes following MCAO. (A) Body weight, shown as a percentage of pre-MCAO weight, decreased the first $2 \mathrm{~d}$ following the MCAO for both groups. The ECA-unligated group (analgesia-treated with no ECA ligation at MCAO) showed a trend toward reduced weight loss on the second day following the MCAO. (B) This panel shows the lesion volume $\left(\mathrm{mm}^{3}\right)$ measured by standard triphenyltetrazolium chloride (TTC) staining $48 \mathrm{~h}$ after the MCAO. The data shown are the mean \pm standard deviation. ECA ligated: $n=17$, ECA unligated: $n=10$. This figure has been modified from Trotman-Lucas et al. ${ }^{11}$. Please click here to view a larger version of this figure. 


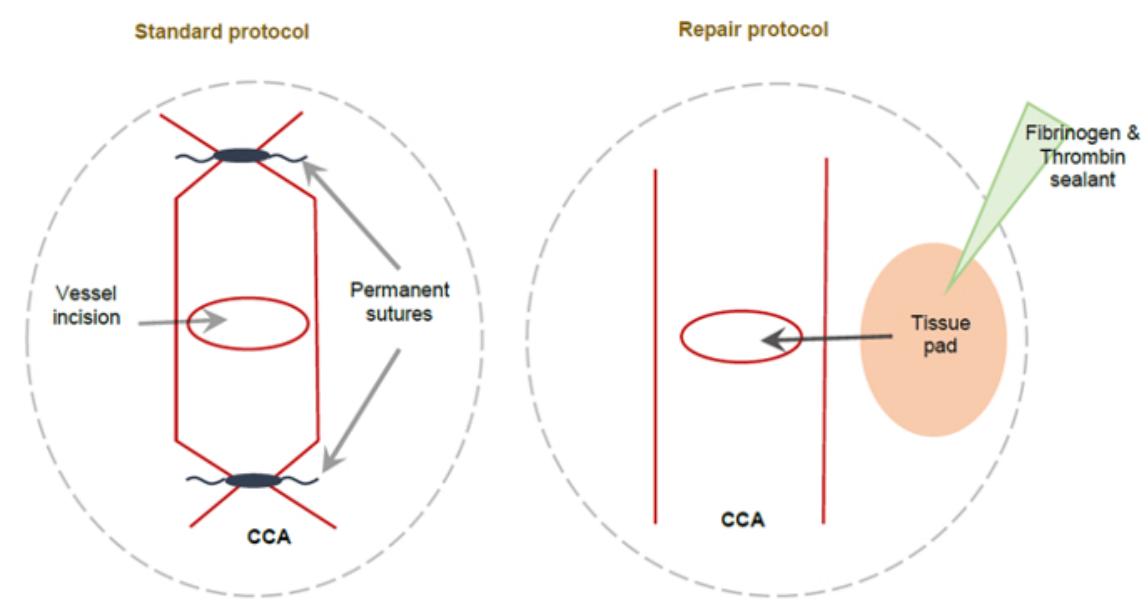

Figure 2: Schematic showing the standard CCA method and the alternative CCA repair method following MCAO. (A) This schematic depicts a permanently ligated CCA using non-dissolvable sutures applied to either side of the CCA incision, resulting in the permanent ligation of the right CCA. (B) This schematic depicts the alternative CCA repair method. A small tissue pad coated with fibrinogen and thrombin sealant is used to cover the CCA incision, sealing it to allow the full perfusion of the right CCA. This figure has been modified from Trotman-Lucas et al. ${ }^{11}$. Please click here to view a larger version of this figure. 


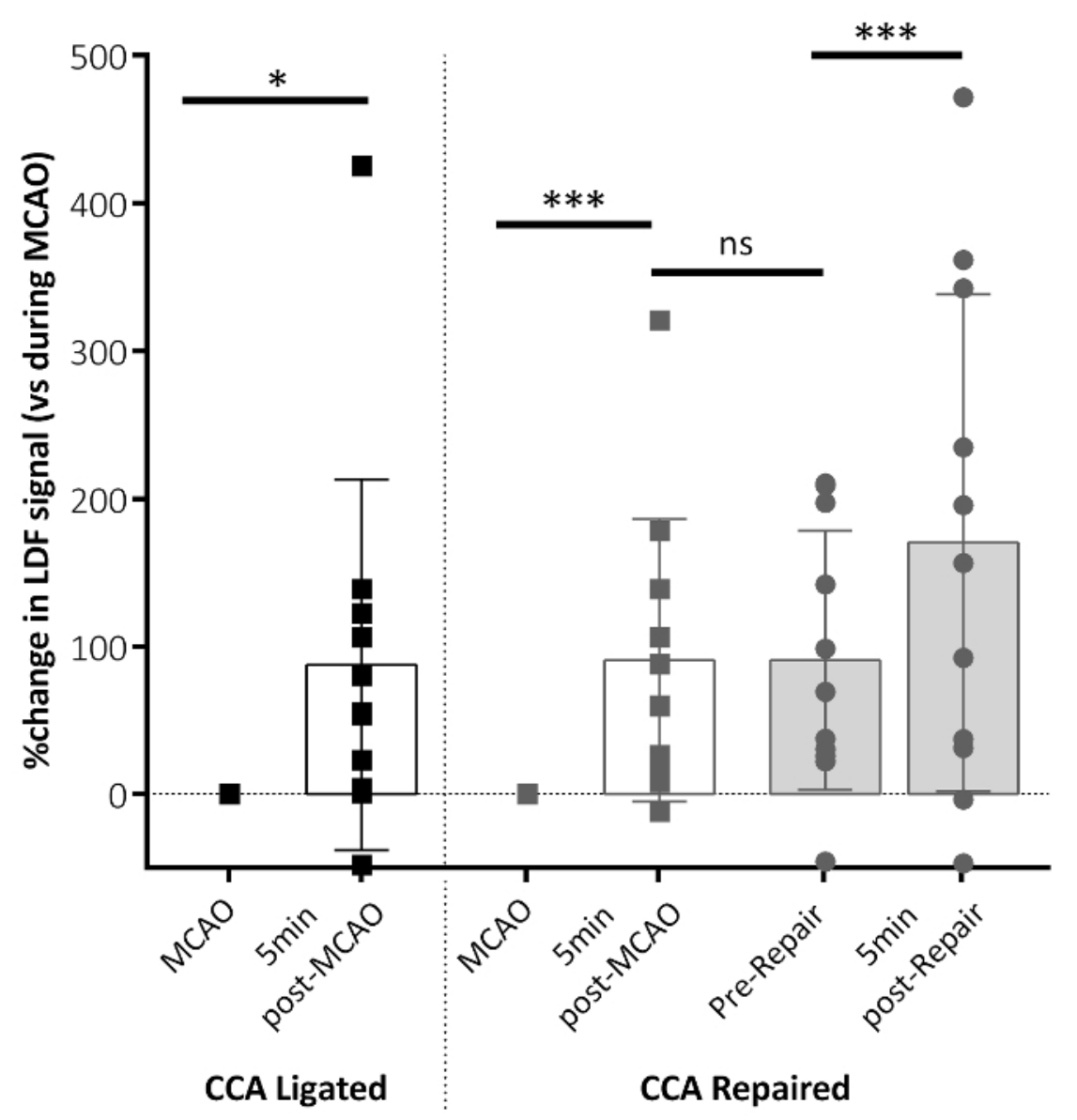

Figure 3: Regional cerebral blood flow (rCBF) parameters following MCAO. The rCBF changed 5 min after the MCAO filament removal for both the CCA-ligated and CCA-repaired groups (post-MCAO), relative to the $\mathrm{CCBF}$ measured during MCAO. This panel shows the rCBF data immediately before the CCA vessel repair (pre-repair) and $5 \mathrm{~min}$ following the CCA repair (post-repair). Significant increases in the rCBF are shown 5 min after the filament removal (post-MCAO) in both groups. An additional increase in the rCBF is shown following the CCA repair (postrepair) in the CCA repair group. No difference in the $\mathrm{rCBF}$ is shown between 5 min post-MCAO and pre-repair. The data shown are condensed from the analyzed time-course data reporting key time points, here as the mean \pm standard deviation. CCA ligated: $n=10$, CCA repaired: $n=10$; ${ }^{* *} P<0.01,{ }^{* *} P<0.001, n s:$ non-significant. This figure has been modified from Trotman-Lucas et al. ${ }^{11}$. Please click here to view a larger version of this figure. 
A

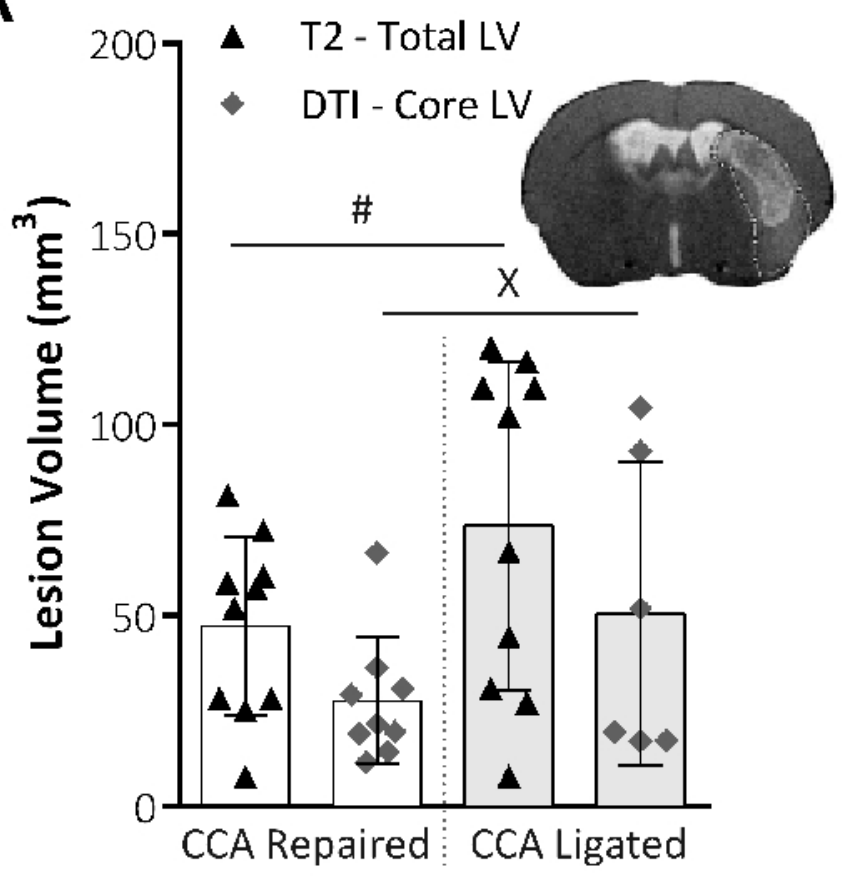

B

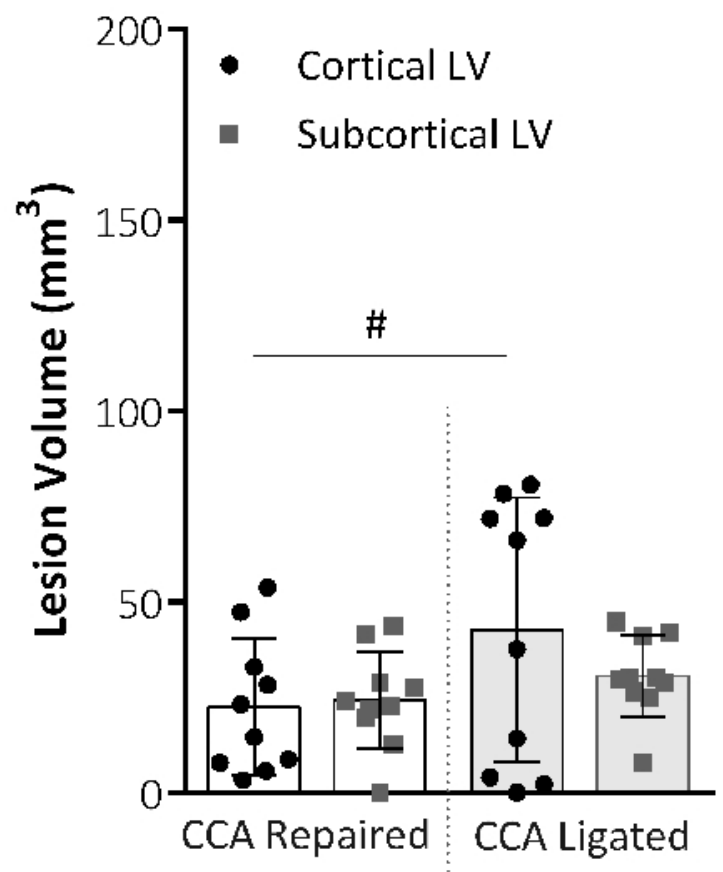

Figure 4: Analysis of the lesion volume obtained by MRI techniques. (A) This panel shows the lesion volume $\left(\mathrm{LV} ; \mathrm{mm}^{3}\right)$ at $48 \mathrm{~h}$ following MCAO, the total LV taken from T2-weighted MRI images (Total LV), and the core LV taken from DTI scans and analysis (Core LV). Representative images show the total lesion volume from a T2 scan slice image with the DTI core lesion volume mask applied. The variability within the groups was significantly reduced for both the total LV $(P=0.015$, CCA repair: $n=10$, CCA ligated: $n=10, F$-test $)$ and the core LV $(P$ $=0.043$, CCA repair: $n=9$, CCA ligated: $n=6$, Lavene's test), assessed using an F-test for parametric data or Levene's test for non-parametric data. (B) This panel shows the LV at $48 \mathrm{~h}$ following MCAO, taken from T2-weighted MRI images and divided into cortical and subcortical lesion areas. The CCA repair significantly reduced the data variability $(P=0.03, F$-test $)$ in the cortical portion of the lesion, but no effect on the data variability in the subcortical portion of the lesion was shown. CCA repair: $n=10$, CCA ligated: $n=10$. The data shown are the mean \pm standard deviation. ${ }^{\#} P<0.05$ (F-test), ${ }^{\mathrm{x}} P<0.05$ (Lavene's test). This figure has been modified from Trotman-Lucas et al. ${ }^{11}$. Please click here to view a larger version of this figure. 


\begin{tabular}{|l|l|l|l|l|l|}
\hline Approach & $\begin{array}{l}\text { Lesion Volume } \\
\text { (LV; } \mathbf{m m}^{3} ; \text { mean } \pm \\
\text { s.d.) }\end{array}$ & Power & Significance Level & $\begin{array}{l}\text { Anticiapted } \\
\text { difference }\end{array}$ & Group size required \\
\hline $\begin{array}{l}\text { CCA Ligated } \\
\text { (traditional approach) }\end{array}$ & $94.08 \pm 53.79$ & 0.8 & 0.05 & $30 \%$ & $n=58$ \\
\hline $\begin{array}{l}\text { CCA Repaired (new } \\
\text { approach) }\end{array}$ & $51.73 \pm 22.78$ & 0.8 & 0.05 & $30 \%$ & $n=35$ \\
\hline
\end{tabular}

Table 1: Representative power analysis comparing traditional CCA ligation with the alternative CCA repair method explained here. This table shows the power analysis conducted to calculate the anticipated group size required to detect a significant difference in the LV between a control group, the traditional or alternative (new) approach, and a test group (predicted). The table shows the group sizes as required if a power of 0.8 is assumed, a significance level of 0.05 is applied, and if the predicted test group shows a $30 \%$ difference in the LV compared to the control group. The table shows results for both MCAO approaches (the CCA-ligated and the CCA-repaired) to determine if there is a difference in the number of animals required to gain a $30 \%$ difference in the LV. For both methods, an equal variance is assumed between the test and the control group. This figure has been modified from Trotman-Lucas et al. ${ }^{11}$.

\section{Discussion}

Filament induction of transient MCAO in rodents is the most frequently used experimental stroke model, as it allows reperfusion to the affected area, mimicking the occurrence of events following clinical ischemic stroke ${ }^{7}$. Reported here is an alternative surgical approach to the traditional method of filament-induced transient MCAO in mice. The alternative approach, involving analgesia treatment, ECA ligation avoidance, and CCA incision repair, results in a reduced LV variability when assessed using both MRI and histological staining methods ${ }^{11}$.

Traditional approaches to induce MCAO largely rely on the transection, or at least ligation, of the ECA, which has been shown, in rats, to affect drinking behavior and an increase in body weight loss following the MCAO ${ }^{14}$. The protocol defined here, in mice, with the avoidance of ECA ligation and analgesia addition, suggested a reduction in body weight loss following the MCAO with no effect on the lesion volume. The use of analgesia is avoided, or at least not reported, in the majority of experimental stroke studies, due to possible confounding effects on the experimental outcome. However, avoiding analgesia completely is not always justified and there is a need to balance the welfare needs of the animals with the achievement of the scientific objectives.

Differences in animal size, strain, and cerebrovascular anatomy, in addition to filament size and type variations, are all suggested to influence stroke outcomes ${ }^{23,24}$. The alternative approach described here avoids the reliance on the CoW during reperfusion, thus reducing, at least in part, the variability seen between animals in lesion volume. CoW anatomy is highly variable in mice, in particular in the $\mathrm{C} 57 \mathrm{BL} / 6 \mathrm{strain}$, which is often used in experimental stroke studies. $90 \%$ of C57BL/6 mice have an incomplete CoW due to a varied posterior communicating artery (PcomA) patency, which may have an effect on the volume of ischemic damage due to the insufficient perfusion of structures outside of the MCA territory ${ }^{13,25}$. Repairing the CCA in mice, as shown here, results in the re-establishment of blood flow via the CCA to the ischemic area, as previously described in rats ${ }^{15}$. The representative data here show that the repair of the CCA increases reperfusion, although the blood flow in the CCA was not directly measured. However, it is possible for the surgeon to visualize the CCA reperfuse with blood following the vessel repair, as it returns to a pulsing and full state all along the trunk, proximal and distal to the repair location. This visual confirmation, along with laser Doppler flowmetry readings of the ischemic area, can be used to confirm the successful repair of the vessel. The time between the tissue pad application and the removal of the vessel clip from the CCA can have an impact on the resulting patency of the CCA, as reducing the time between the tissue pad application and the clip removal will prevent the tissue pad from adhering to the opposite side of the CCA. Although technically challenging, the alternative MCAO procedure explained here does not require any additional skills than those required to perform the surgical induction of MCAO in mice.

Traditionally associated with a high variability in outcome measures, experimental stroke studies may have a tendency to be underpowered. Ethical and welfare requirements in combination with economic and practical concerns may contribute to studies being underpowered. By reducing the variability in outcome and, therefore, producing more consistent lesion outcomes across an experimental group, more effective power calculations can be performed with the ultimate aim of studies being appropriately powered.

In conclusion, this alternative CCA repair procedure, in mice, results in less variability in lesion volume following experimental stroke and enables smaller experimental groups to be required for testing a treatment effect when appropriate power calculations are used.

\section{Disclosures}

The authors have nothing to disclose.

\section{Acknowledgments}

This work was funded by the National Centre for the Replacement, Refinement and Reduction of Animals in Research (NC3Rs; NC/M000117/1 to CG). The authors thank the staff of the Division of Biomedical Services, University of Leicester, for their care of the experimental animals and Maria Viskaduraki for her statistical advice. The representative results are adapted with permission from Disease Models $\&$ Mechanisms ${ }^{11}$. 


\section{References}

1. O'Collins, V. E. et al. 1,026 Experimental treatments in acute stroke. Annals of Neurology. 59 (3), 467-477 (2006).

2. Sutherland, B. A. et al. Neuroprotection for Ischaemic Stroke: Translation from the Bench to the Bedside. International Journal of Stroke. 7 (5), 407-418 (2012).

3. Reeves, M. J. et al. Acute Stroke Care in the US: Results from 4 Pilot Prototypes of the Paul Coverdell National Acute Stroke Registry. Stroke. 36 (6), 1232-1240 (2005).

4. Wardlaw, J. M., Murray, V., Berge, E., del Zoppo, G. J. Thrombolysis for acute ischaemic stroke. Cochrane Database of Systematic Reviews. (7), CD000213 (2014).

5. Pangalos, M. N., Schechter, L. E., Hurko, O. Drug development for CNS disorders: strategies for balancing risk and reducing attrition. Nature Reviews Drug Discovery. 6 (7), 521-532 (2007).

6. Hossmann, K. A. Pathophysiological basis of translational stroke research. Folia Neuropathologica. 47 (3), $213-227$ (2009).

7. Ringelstein, E. B. et al. Type and extent of hemispheric brain infarctions and clinical outcome in early and delayed middle cerebral artery recanalization. Neurology. 42 (2), 289-289 (1992).

8. Carmichael, S. T. Rodent models of focal stroke: Size, mechanism, and purpose. NeuroRX. 2 (3), 396-409 (2005).

9. Dirnagl, U. Bench to Bedside: The Quest for Quality in Experimental Stroke Research. Journal of Cerebral Blood Flow \& Metabolism. 26 (12), 1465-1478 (2006).

10. Ingberg, E., Dock, H., Theodorsson, E., Theodorsson, A., Ström, J. O. Method parameters' impact on mortality and variability in mouse stroke experiments: a meta-analysis. Scientific Reports. 6, 21086 (2016).

11. Trotman-Lucas, M., Kelly, M. E., Janus, J., Fern, R., Gibson, C. L. An alternative surgical approach reduces variability following filament induction of experimental stroke in mice. Disease Models \& Mechanisms. 10 (7), 931-938 (2017).

12. Macrae, I. M. Preclinical stroke research - advantages and disadvantages of the most common rodent models of focal ischaemia. British Journal of Pharmacology. 164 (4), 1062-1078 (2011).

13. McColl, B. W., Carswell, H. V., McCulloch, J., Horsburgh, K. Extension of cerebral hypoperfusion and ischaemic pathology beyond MCA territory after intraluminal filament occlusion in C57BI/6J mice. Brain Research. 997 (1), 15-23 (2004).

14. Trueman, R. C. et al. A Critical Re-Examination of the Intraluminal Filament MCAO Model: Impact of External Carotid Artery Transection Translational Stroke Research. 2 (4), 651-661 (2011).

15. Dittmar, M. S. et al. The role of ECA transection in the development of masticatory lesions in the MCAO filament model. Experimental Neurology. 195 (2), 372-378 (2005).

16. Kilkenny, C., Browne, W. J., Cuthill, I. C., Emerson, M., Altman, D. G. Improving Bioscience Research Reporting: The ARRIVE Guidelines for Reporting Animal Research. Plos Biology. 8 (6), e1000412 (2010).

17. Langford, D. J. et al. Coding of facial expressions of pain in the laboratory mouse. Nature Methods. 7 (6), $447-479$ (2010).

18. Orsini, F. et al. Targeting Mannose-Binding Lectin Confers Long-Lasting Protection With a Surprisingly Wide Therapeutic Window in Cerebral Ischemia. Circulation. 126 (12), 1484-1494 (2012).

19. Simoni, M. G. D. et al. Neuroprotection by Complement (C1) Inhibitor in Mouse Transient Brain Ischemia. Journal of Cerebral Blood Flow \& Metabolism. 23 (2), 232-239 (2003).

20. Clark, W., Gunion-Rinker, L., Lessov, N., Hazel, K. Citicoline Treatment for Experimental Intracerebral Hemorrhage in Mice. Stroke. 29 (10), 2136-2140 (1998).

21. Lin, T. N., He, Y. Y., Wu, G., Khan, M., Hsu, C. Y. Effect of Brain Edema on Infarct Volume in a Focal Cerebral-Ischemia Model in Rats. Stroke. 24 (1), 117-121 (1993).

22. Loihl, A. K., Asensio, V., Campbell, I. L., Murphy, S. Expression of nitric oxide synthase (NOS)-2 following permanent focal ischemia and the role of nitric oxide in infarct generation in male, female and NOS-2 gene-deficient mice. Brain Research. 830 (1), 155-164 (1999).

23. Connolly, E. S., Winfree, C. J., Stern, D. M., Solomon, R. A., Pinsky, D. J. Procedural and strain-related variables significantly affect outcome in a murine model of focal cerebral ischemia. Neurosurgery. 38 (3), 523-531 (1996).

24. Barone, F. C., Knudsen, D. J., Nelson, A. H., Feuerstein, G. Z., Willette, R. N. Mouse Strain Differences in Susceptibility to Cerebral Ischemia are Related to Cerebral Vascular Anatomy. Journal of Cerebral Blood Flow \& Metabolism. 13 (4), 683-692 (1993).

25. Kitagawa, K. et al. Cerebral Ischemia after Bilateral Carotid Artery Occlusion and Intraluminal Suture Occlusion in Mice: Evaluation of the Patency of the Posterior Communicating Artery. Journal of Cerebral Blood Flow \& Metabolism. 18 (5), $570-579$ (1998). 